\begin{tabular}{|c|c|}
\hline Title & Telemedicine by mobile communication \\
\hline Author(s) & Shimizu, K. \\
\hline Citation & $\begin{array}{l}\text { IEEE Engineering in Medicine and Biology Magazine, 18(4), 32-44 } \\
\text { https://doi.org/10.1109/51.775487 }\end{array}$ \\
\hline Issue Date & 1999-08 \\
\hline Doc URL & http:/hdl .handle.net/2115/5579 \\
\hline Rights & $\begin{array}{l}\text { IEEE, Engineering in Medicine and Biology Magaziner, } 18,4,1999, \text { p32-44 } \\
\text { @1999 IEEE. Personal use of this material is permitted. However, permission to reprint/republish this material for } \\
\text { advertising or promotional purposes or for creating new collective works for resale or redistribution to servers or lists, } \\
\text { or to reuse any copyrighted component of this work in other works must be obtained from the IEEE." }\end{array}$ \\
\hline Type & article \\
\hline File Information & IEMBM18-4.pdf \\
\hline
\end{tabular}

Instructions for use 


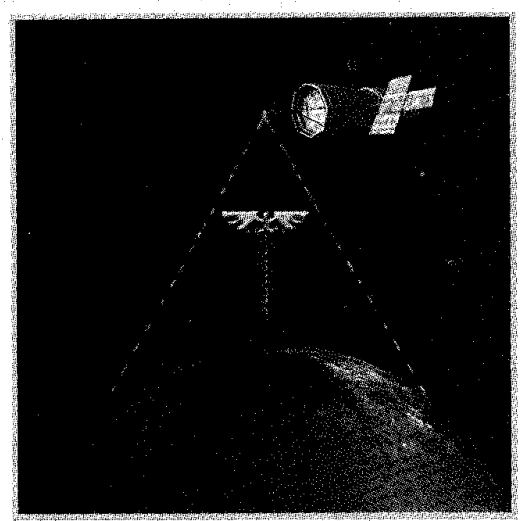

Koichi Shimizu

Department of Biological Systems Engineering,

Graduate School of Engineering

Hokkaido University, Sapporo

\section{Telemedicine by Mobile Communication}

\section{Techniques for Multiple Data Transmission from Moving Vehicles in Emergency Medicine Situations}

\begin{abstract}
here have been many studies that have proved the feasibility and usefulness of telemedicine [1-3]. Remote diagnosis of an ECG through a telephone network is a well-known example [4]. Telemedicine is a useful technique and has been used in such applications as medical care in remote rural areas and aftercare for pacemaker-implanted patients. However, the use of telemedicine has not spread as extensively as other commonly used engineering techniques, such as medical imaging. This is not because telemedicine is of less importance, but because supporting technologies have not been sufficiently developed. Recently, however, the situation has changed due to progress in communication technology. The performance of communication links has greatly improved, and a communication network has been extended throughout most of the world. Mobile communication and satellite communication, in particular, have brought about new possibilities in various fields. Applications of these techniques are expected to open new horizons in the field of telemedicine, as well.
\end{abstract}

It has been recognized that telemedicine is useful in the field of emergency medicine. Early diagnosis by a specialist in an ambulance is particularly important for emergency care $[5,6]$. Moreover, recent social changes in Japan (i.e., an increasing population of senior citizens and increasingly stressful living conditions) have resulted in a rapid increase of acute cardiovascular and cerebrovascular crises, which have, in turn, increased the demand for telemedicine. People are also spending longer periods of time in moving vehicles; for example, in aircraft on an international route and in ships plying the ocean. Thus, the number of emergency cases in such situations has steadily increased. However, at present, there are no effective means for coping with emergency cases on moving vehicles. In such emergencies, accurate diagnosis is one of the most important factors for the survival and aftercare of the patient.

Some projects have been conducted in which the techniques of mobile communication and satellite communication were applied to telemedicine. Early experiments were conducted with the transmis-

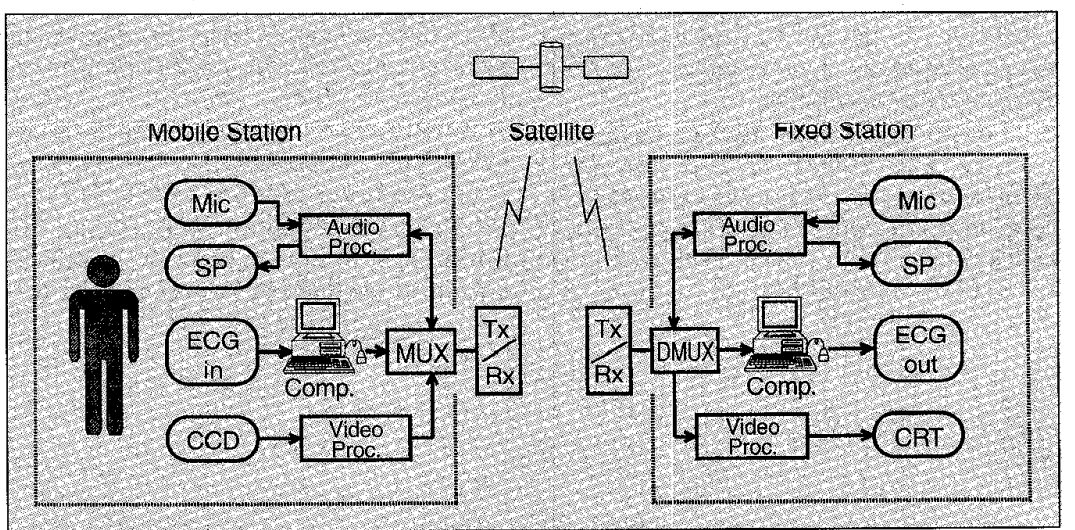

1. Principle of telemedicine using mobile satellite communication. 
sion of the ECG from an ambulance $[5,6]$, and with a medical consultation service transmitted by a communication satellite [7]. Although the feasibility has been verified, the transmission of such medical information has not been widely practiced in most countries.

We have proposed some techniques for mobile telemedicine and have verified their practical feasibility in experiments. This article presents technical considerations required to realize a practical mobile telemedicine system, techniques developed for multiple medical data transmission, and the satisfactory results of their applications to telemedicine in moving vehicles.

\section{Technical Considerations for Mobile Telemedicine}

We have proposed the application of biotelemetry techniques to telemedicine using mobile communication [8], as illustrated in Fig. 1. In a moving vehicle, color images, audio signals, and physiological signals such as ECG and blood pressure are obtained from the patient. These images and signals are multiplexed and transmitted to a fixed station. In the fixed station, the signals received are demultiplexed and presented to a medical doctor, or processed in an automatic monitoring system. Instructions from the doctor are then transmitted back to the mobile station through the communication link.

This technique has inherent characteristic problems that are different from those of conventional telemedicine or general data transmission using mobile communication. In the following sections these problems are pointed out and technical solutions are presented to show the practical feasibility of the proposed technique.

\section{Channel Capacity}

In mobile communication, the capacity of the transmission link is generally limited. It is typically $10 \mathrm{kbps}-100 \mathrm{kbps}$. For example, cellular telephones in Japan have a transmission capacity of 9.6-64 $\mathrm{kbps}$, and the digital link of INMARSATs has a transmission capacity of 24-64 kbps. These capacities are far below those required for the transmission of medically significant information, such as a moving color image. The transmission of a color video signal requires a transmission capacity of in the order of $1-10 \mathrm{Mbps}$ with data compression for a moving image. Thus, we must first examine whether any useful medical data transmission can be performed with such limited capacity. For practical telemedicine using this limited transmission capacity, the following parameters were chosen and data-compression techniques were applied. Table 1 shows an example of the parameters and data-reduction ratios used.

In emergency telemedicine, visual information is of vital importance [9]. In some cases, high-quality colored images are required to check the color of skin, lips, nails and blood, as well as the condition of the patient's body. In other cases, moving images are required to check the movement of a patient. In the former case, still pictures are transmitted. By making the interval between each picture short (e.g., less than $1 \mathrm{~min}$ ), slow movements of the patient can be understood. In the latter case, if a picture with low image quality is sufficient, a monochromatic moving picture is transmitted. Various techniques have been developed to compress image data. The technique considered in this study is based on the vector quantification of differential luminance and chrominance signals [10]. With this technique, 10:1 compression is possible, and color still-pictures can be transmitted every 20 $\mathrm{sec}$ at $8 \mathrm{kbps}$.

Audio signals are used primarily for communication between a doctor and a patient or crewmembers. Intelligibility is important, but an extremely high quality of sound is not required. Thus, in this study, the audio signal is bandlimited from 1.5 to $3 \mathrm{kHz}$, and an adaptive delta modulation technique [11,12] is applied with a sampling frequency of $6 \mathrm{kHz}$. This allows a compression ratio of $4.8: 1$ and a transmission rate of $10 \mathrm{kbps}$.

For the biological signals to be transmitted, ECG and blood pressure were chosen as fundamental variables to indicate vital signs. To transmit three-channel ECG signals, a channel capacity of 4.8 kbps is required. Many techniques have been developed for ECG data compression [13], and it is not difficult to reduce such data by $8: 1$.

Systolic and diastolic blood pressures are measured every minute. Since the dynamic range and sampling rate are small, only a low bit rate and no compression are required

The total capacity required for transmitting all of this data is about $19 \mathrm{kbps}$. Therefore, even if more capacity is required for other signals to provide multiplexing and redundancy (e.g., synchronization bits, parity bits, etc.), all these data can be trans-

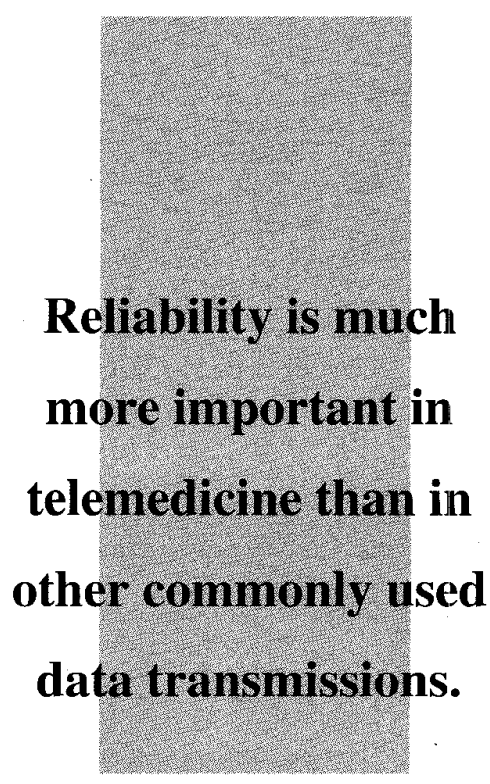

mitted within the practical capacity of a mobile communication link.

\section{Reliability}

In telemedicine, a transmission error can cause a fatal outcome. Therefore, reliability is much more important in telemedicine than in other commonly used data transmissions. A high level of reliability can be obtained by introducing as much redundancy as possible in the signal transmission; e.g., by making the handshake transmission process complex, and by establishing strict error-checking or error-control. However, such measures are difficult with the aforementioned low transmission capacity. If we use a satellite link, a significant propagation delay (about $0.5 \mathrm{sec}$ ) becomes another problem.

In this study, the following error-control techniques were applied. An automatic repeat request (ARQ) tech-

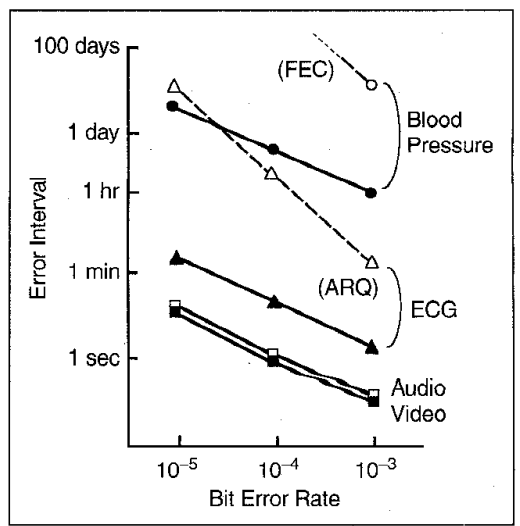

2. Theoretical analysis of reliability and improvements by error-control techniques. 
nique was employed for the ECG. Although ARQ is highly reliable in data transmission, retransmission of data produces a delay when an error is found. This problem can be solved by the technique described below. There is no problem with retransmission if we use the method of forward error correction (FEC). We employed FEC for blood pressure, and error-control was conducted according to the majority decision principle.

The reliability of each parameter was analyzed theoretically. First, an error interval (EI), or a mean time between errors, is defined as:

$$
E I=L_{\text {data }} /\left(E_{\text {data }} R_{\text {data }}\right)
$$

where $E_{\text {data }}$ is the probability that the data is in error, $\mathbf{L}_{\text {data }}$ is the data length [bit], and $R_{\text {data }}$ is the data rate $[\mathrm{bit} / \mathrm{sec}] . \mathrm{E}_{\text {data }}$ depends on the coding method of the data.

In our system, EIs are theoretically calculated for ECG and blood pressure. The results are shown in Fig. 2. The bit-error rate (BER) is assumed to be $10^{-3}$ in the worst possible case, which seldom happens. The BER of the communication link used in this study was about $10^{-5}$ in the usual situation. Thus, as can be seen in the figure, sufficient reliability is attained. with the error control.

EIs are calculated for the image and audio signals in a similar way. An error in these data is produced once every 5-50 sec. Therefore, an error appears in an image within this interval, but it is only in a small part of the image and can thus be ignored. In the case of audio data, errors occur over a short period of time and do not affect the intelligibility of the conversation. The above data are presented to the human visual and auditory sensing systems, where considerable error can be tolerated from the viewpoint of recognition. Thus, sufficient reliability is guaranteed in this system.

\section{Real-Time Operation}

In telemedicine, real-time communication between moving and fixed stations is important. Recently, the operation speeds of microprocessors and communication control units have increased greatly. This has made real-time data transmission easier, even with complex error-control procedures. However, if we use a satellite communication link, the propagation delay between the transmitter and receiver becomes a problem. If ARQ is used as the error-control principle, a delay is inevitable due to the time required for repeat-request signal transmission and data retransmission (Fig. 3(a)).

This problem can be solved by installing input and output buffer memories in both moving and fixed stations. The transmission time has to be made sufficiently shorter than the time corresponding to the buffer capacity. Figure 3 illustrates an example of the delay in the ARQ process and the solution using buffers. Since the speed of data transmission is faster than the speed of inputting and outputting of the original signal, there is no interruption in the output data.

As an example, transmission delay is evaluated in the case of a satellite communication link or a 24 -kbps MSK (minimum shift keying) link. If the BER and the

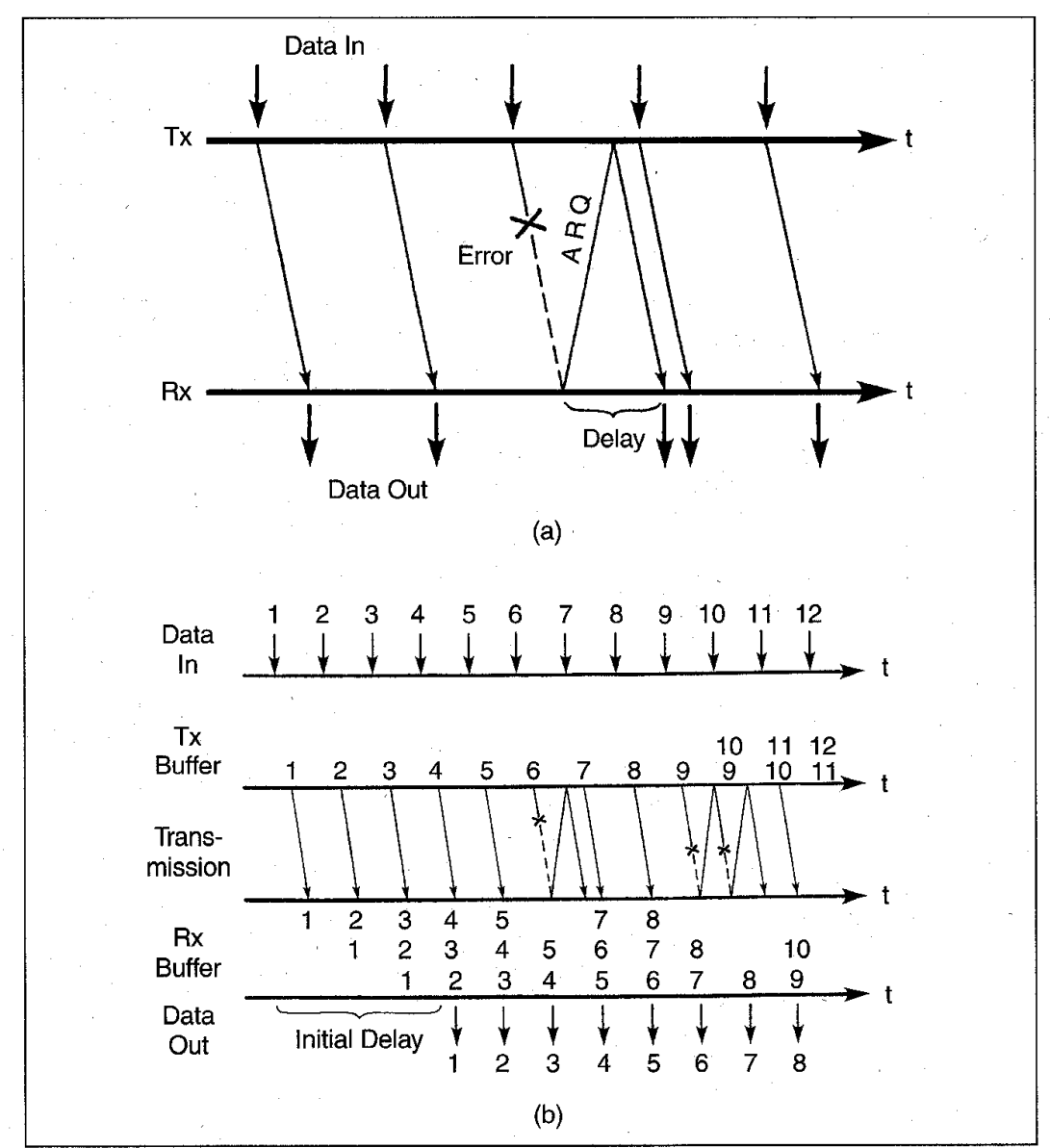

3. Real-time transmission with the ARQ technique. (a) Delay due to retransmission. (b) Buffering for the transmission without interruption.

\begin{tabular}{|l|l|l|l|}
\hline \multicolumn{5}{|c|}{ Table 1. Transmiting Information and Data Compression } \\
\hline Data & Sampling & Compression Ratio & Bit Rate \\
\hline Video & $\begin{array}{l}256 \times 256 \mathrm{pel} / \mathrm{plane} \\
8-\mathrm{bit} \mathrm{RGB/pel} \\
1 \mathrm{plane} / 20 \mathrm{sec}\end{array}$ & $10: 1$ & $8 \mathrm{kbit} / \mathrm{sec}$ \\
\hline Audio & $\begin{array}{l}8 \mathrm{bit} / \mathrm{sample} \\
6000 \text { sample/sec }\end{array}$ & $4.8: 1$ & $10 \mathrm{kbit} / \mathrm{sec}$ \\
\hline ECG & $\begin{array}{l}3 \mathrm{channel} \\
8 \mathrm{bit} / \mathrm{sample} \\
200 \text { sample/sec }\end{array}$ & $8: 1$ & $\begin{array}{l}600 \mathrm{bit} / \mathrm{sec} \\
(3 \mathrm{channel})\end{array}$ \\
\hline Blood Pressure & $\begin{array}{l}16 \mathrm{bit} / \mathrm{sample} \\
1 \mathrm{sample} / \mathrm{min}\end{array}$ & $1: 1$ & $0.3 \mathrm{bit} / \mathrm{sec}$ \\
\hline
\end{tabular}


round-trip delay of the link are $10^{-3}$ and 0.6 $\mathrm{sec}$, respectively, the mean delay is 94 bits or $0.073 \mathrm{sec}$. These are sufficiently small to cope with by the technique mentioned above. Therefore, the real-time operation is not impeded by the propagation delay.

Electromagnetic Interference (EMI)

The electromagnetic environment in a moving vehicle may be adversely affected by ignition noises, radio communication, and radar equipment. Therefore, medical devices and instruments should be designed to be immune to such an unfavorable environment. At the same time, they must not interfere with the navigation equipment of the vehicle, particularly in an airplane or a spaceship.

Regarding electric lines and cables, techniques have been established to suppress both conduction and emission noises. Thus, equipment used in emergency care does not generally have the problem of EMI. However, telemetry devices using radio waves are of primary concern. Although telemetry devices are useful for measuring and monitoring physiological parameters of the patient, they are vulnerable to EMI and can also be the cause of EMI.

A telemetry technique using indirect light transmission [14] is one solution to the above problem. This technique is useful in an enclosed environment, such as the cabin of an airplane, ship, or space-lab. Ambulatory measurement using indirect light transmission has been shown to be feasible, and its effectiveness was verified by the acquisition of physiological data from astronauts [15].

\section{Multiple Data Transmission} Analog Multiplexing

An ECG is one of the most important of the vital signs to be transmitted in emergency care. ECG transmission using a telephone line has been practiced in various applications, such as telemedicine in a remote area and periodic checkups of patients with implanted pacemakers. We have developed a new technique for simultaneously transmitting an ECG and voice through a single vocal communication channel of a mobile telephone or an emergency radio system [16].

This technique enables us to transmit an ECG and vocal messages from an ambulance to a hospital in real time, and to send back instructions from a medical doctor to an ambulance, all in a single vocal channel. It does not require installation of an additional communication channel for the ECG transmission. Figure 4 illustrates the outline of this system. Since real-time operation is required for ECG transmission as well as for vocal communication, frequency-domain multiplexing is used. The major spectral power of the human voice is confined to within the range of $0.4-0.7 \mathrm{kHz}$. We have reported that it is possible to limit the bandwidth for vocal communication to within 0.3-1 $\mathrm{kHz}$ for practical conversation [16].
Based on this analysis, we allotted the lower $(0.3-1.1 \mathrm{kHz})$ and the upper $(2.0$ $2.8 \mathrm{kHz}$ ) frequency ranges for AM vocal signals and FM ECG signals, respectively. The details of this system and its performance have been described elsewhere [16].

\section{Digital Multiplexing of Vital Signs}

For one-channel ECG transmission, the analog multiplexing mentioned above is useful. However, more physio-

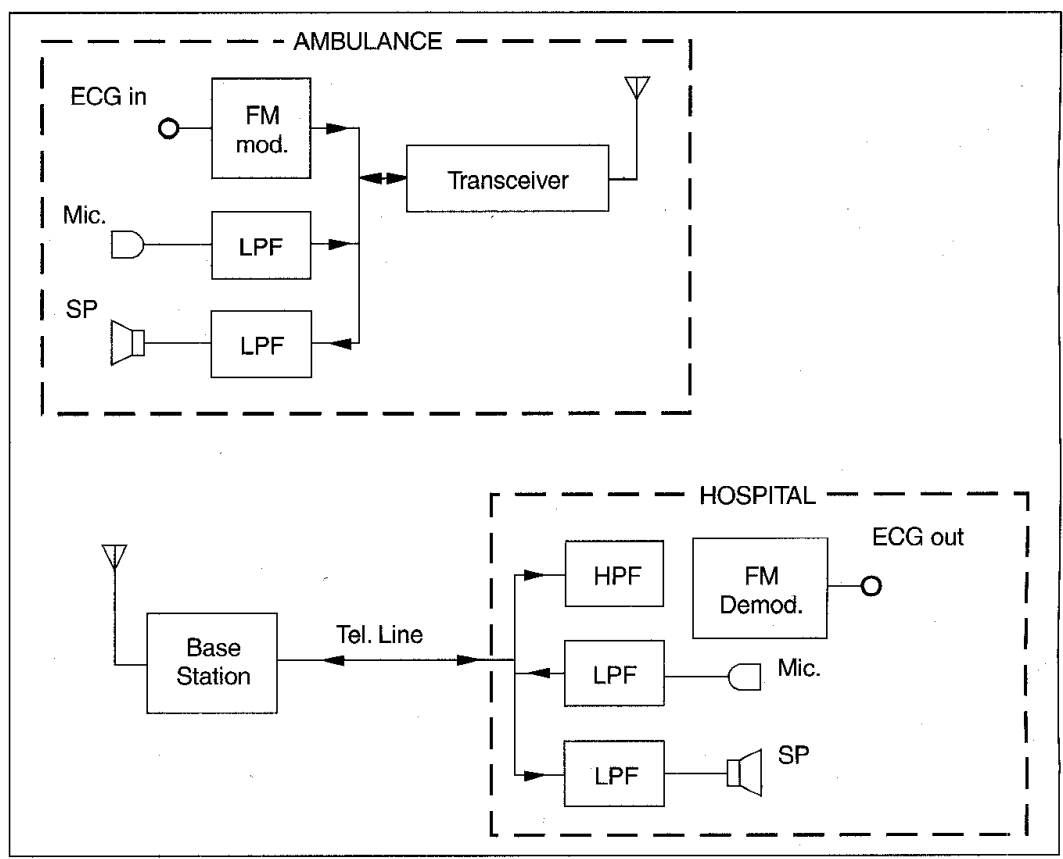

4. Outline of the system for multiplexed transmission of ECG and voice.

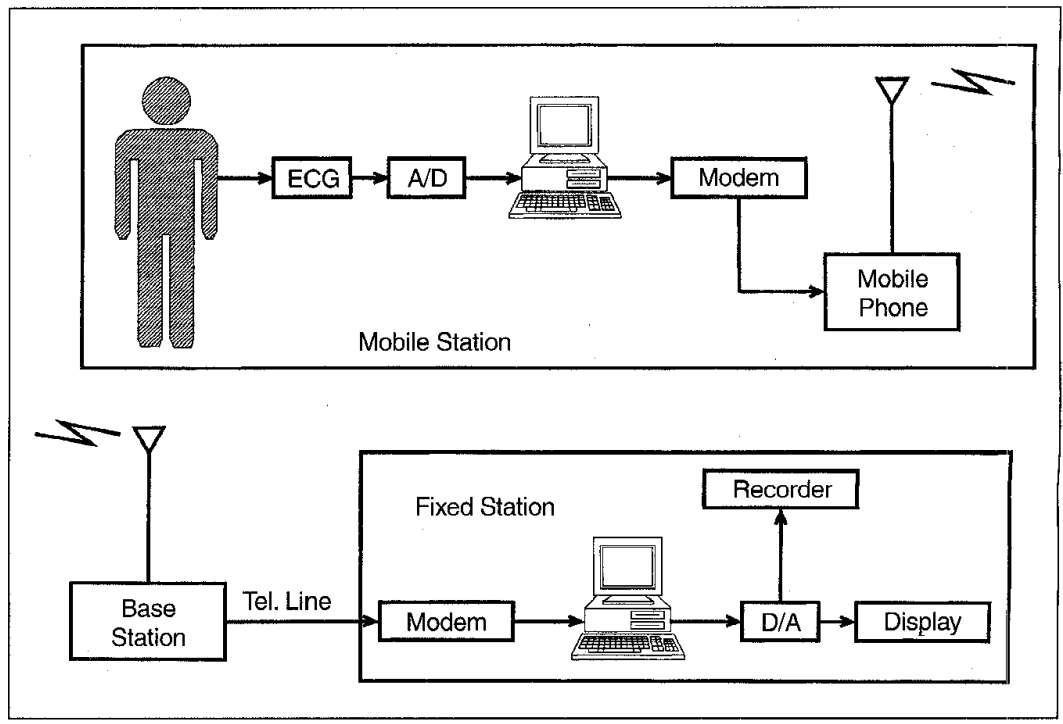

5. Outline of the digital ECG transmission system.

IEEE ENGINEERING IN MEDICINE AND BIOLOGY 
logical information, such as multiple-channel ECG, blood pressures, and blood oxygen level, is sometimes required in emergency medicine. To accommodate multichannel transmission of different kinds of signals, we have developed a digital data-transmission system, as shown in Fig. 5. The vital sign signals are fed into a computer through a transducer and an $\mathrm{A} / \mathrm{D}$ converter. In the computer, the signals are transformed into numerical data according to a predetermined data format. The data are then fed into a modem through an RS $232 \mathrm{C}$ interface. The data are transmitted along the following path: modem to mobile communication link to relay station to wire telephone line to modem. Signals from the receiver modem are fed into a personal computer and vital signs are presented on a CRT display or a recorder through a D/A converter.

The fixed bit-rate allocation method was used as the format for transmitting data. This method is suitable for serial real-time transmission and has a small out-of-synchronization probability. Fundamental analysis for this transmission has been reported elsewhere [8,17]. In this method, the data length of one frame of the serial data must be determined. Some of the bits in a frame, such as parity bits, are used for frame synchronization and for error control. The shorter the data length in a frame is, the less transmission error can be expected. However, if the data length in a frame is short, the throughput of the data becomes worse. Error analysis was conducted to find the optimal frame length in this tradeoff between reliability and efficiency. The bit allocation for different kinds of data in a frame of 1,200 bits is shown in Table 2 .

\section{Telemedicine in an Ambulance} Fundamental Principle

Figure 6 illustrates the principle of telemedicine in an ambulance [18]. The appearance of the patient is taken by a CCD camera at the accident scene or in the ambulance. Color still-pictures are transmitted, and each scene is renewed every $20-80 \mathrm{sec}$. Vital signs, such as an ECG and blood pressure, are sampled, digitized, and multiplexed in a personal computer. The signals are fed into the transmitter through a modem, and then transmitted to a central fire station using an emergency radio link or a mobile telephone network.

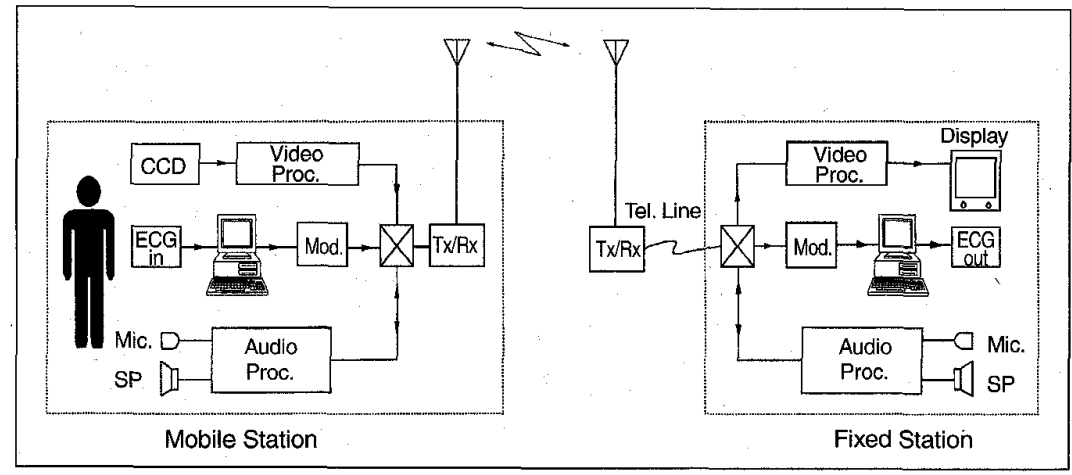

6. Principle of multiple medical data transmission from a moving ambulance.

At the fire station, the communication channel is connected to a telephone line, and the signals are transferred to an appropriate hospital. In the hospital, a medical doctor can view the color images and monitor the patient's vital signs in real time. The doctor then sends the necessary. information to the paramedics through a vocal communication channel.

\section{Data-Transmission Media}

The emergency radio link of a fire station and a mobile telephone network are used as wireless transmission media. In the former case, the signals from an ambulance are transmitted to a relay station on the top of a mountain in the suburbs of our city (Sapporo) using a radio frequency ( $150 \mathrm{MHz}$ band FM, $10 \mathrm{~W}$ ). The signal is then transmitted from the relay station to the central operating room of the fire station through a microwave network of 12 $\mathrm{GHz}$. Based on the judgement of the fire-station operator, the signal channel is connected to a wire telephone network. In this way, the signal is transmitted to output devices such as a CRT display or an ECG recorder in the emergency room of a hospital.

In the case of a mobile telephone network, signals are transmitted by radio waves ( $900 \mathrm{MHz}$ band) from the ambulance to the nearest base station of the mo-

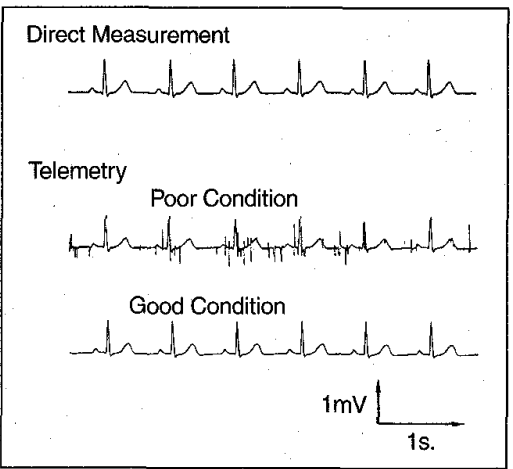

7. Results of analog ECG tranșmission from a moving car.

bile telephone system. The wireless channel is automatically connected at the base station to the wire telephone network. The signals are then transmitted to the telephone terminal in an emergency room of a hospital.

\section{Multiplexed Transmission of Analog ECG}

Using the experimental system described above, experiments were conducted on simultaneous transmission of, an analog ECG and a vocal message from a moving car. More than $90 \%$ of the test sentences, which had no connection with each other, were clearly understood. If the sentence was not clear in practice, the receiving person could ask for the sentence

\begin{tabular}{|l|l|l|}
\hline \multicolumn{3}{|c|}{ Table 2. Designed Frame Structure and Transmission Rates } \\
\hline Data & Rate (kbit/sec) & Length (bit/frame) \\
\hline Video & 11.36 & 568 \\
\hline Audio & 10.08 & 504 \\
\hline ECG & 1.28 & 64 \\
\hline Blood Pressure & 0.02 & 1 \\
\hline Others & 1.26 & 63 \\
\hline Total & 24.00 & 1200 \\
\hline
\end{tabular}




\section{When the}

\section{communication link}

\section{was not good, noise}

\section{appeared in the}

\section{received $\mathrm{ECG}$, but the}

major waveform could

still be recognized.

to be repeated. Overall, there were no serious problems found, and we considered this system to be sufficient for practical operations [16].

To examine the usefulness of this system, experiments were conducted on transmission of an ECG from a moving ambulance. In the central city and suburban areas, the results were satisfactory except for when the ambulance passed through a tunnel or an underpass. Figure 7 shows an example of the results. When the communication link was not good, noise appeared in the received ECG. However, the major waveform of an ECG could still be recognized in a noisy signal. Figure 8 shows a typical example of temporal changes in the received signal quality. The ordinate is the correlation coefficient between the input ECG waveform of a transmitter and the output waveform of a receiver. Generally, the ECG waveform is sufficient for clinical diagnosis if the correlation coefficient is more than $0.8-0.9$. As can be seen in Fig. 8(a), the results using a mobile telephone line were satisfactory. The temporal degradation of signal quality at 10 and 50 min after the start of transmission seemed to be due to the hand-off phenomenon of a mobile telephone line. In the case of an emergency radio link (Fig. 8(b)), the degradation of signal transmission was relatively large, especially from 15 to 50 min after the start of transmission.
To investigate the cause of this degradation, the strength of the received radio wave was analyzed. Figure 9 shows a comparison between the received level of the radio wave and the correlation coefficient of the input and output ECG waveforms. The figure shows that a decrease in the reception level is a major cause of the degradation of signal quality. This means that even in our experimental system, a reasonable quality of signal transmission can be expected as long as the reception level of the radio wave is maintained appropriately. This expectation was supported by the results of the latter half of the experiment (Fig.8(b)). The steep drops in signal quality at around 80 and 130 min were due to interruptions in the communication link when the ambulance went through an underpass.

\section{Multichannel Digital ECG Transmission}

An experiment on digital ECG transmission was conducted using the system described above. Figure 10 shows an ECG transmitted by a regular modem for a mobile telephone. When the car was stopped and the communication link was stable, the quality of the received signal was good. However, when the car was moving, interruption and delay in the ECG waveform were observed. The delay sometimes exceeded several tens of seconds, which is not acceptable for emergency medicine.

The cause of the delay was the error-free operation of the modem used in the experiment. The modem was originally designed for mobile digital data transmission, such as a facsimile machine in a car. Thus, the modem at the receiving end kept requiring the repeated transmission of the data until intact data were received. The effective transmission rate thus became lower than the input and output data rate. This resulted in the delay seen in Fig.10, and eventually some data were lost when the transmitter buffer overflowed. We call transmission using this modem "error-free mode."

To solve this transmission problem, a different type of modem was used. We call transmission using this modem "intelligent mode." With this intelligent modem, the effective data-transmission rate was significantly improved by the use of a data-compression technique. The software for data transmission was also redesigned to renew old data in the buffer if

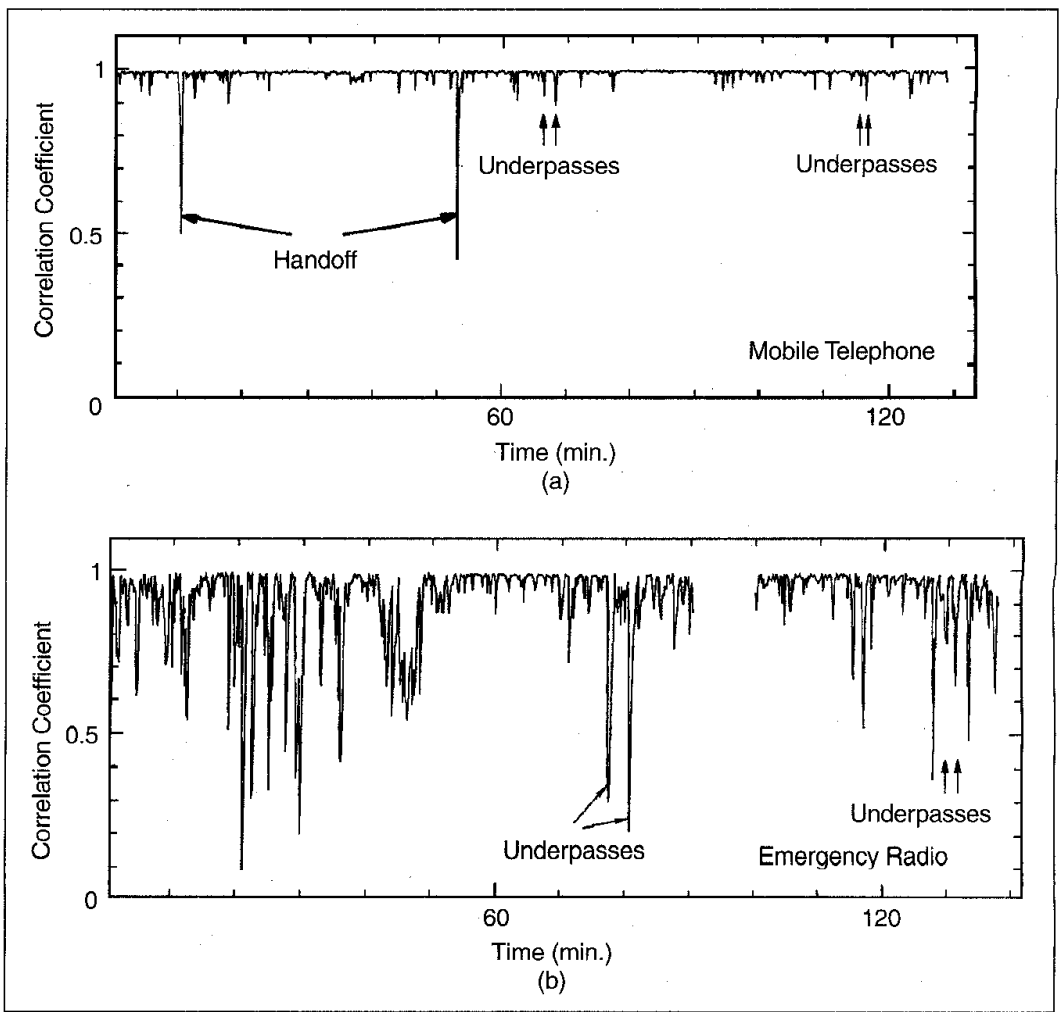

8. Temporal changes in the quality of received signals in analog ECG transmission: (a) using a mobile telephone, (b) using an emergency radio. 
buffer-overflow occurred. Thus, even if the communication link is cul by more than the buffering time, the latest ECG signals are received almost instantly when the link is recovered. In this way, real-time transmission of an ECG was maintained under an error-control protocol.

Figure 11 shows the results of an ECG transmission in the intelligent mode. When the car was running in its usual manner, the quality of the ECG transmission was high. When the car passed through a tunnel, the communication link was temporarily cut and the ECG signal was lost for a short time, but real-time transmission was resumed when the car came out of the tunnel. In the experiment, the delay between the input signal to the

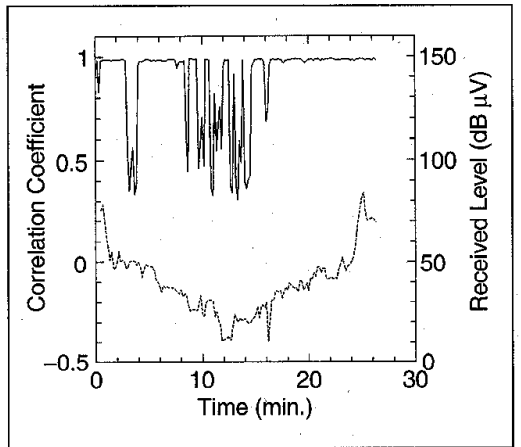

9. Comparison of received signal quality (solid line) and received level of radio wave (dashed line).

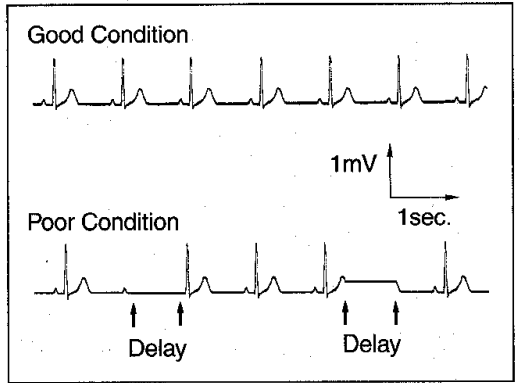

10. Results of digital ECG transmission in error-free mode.

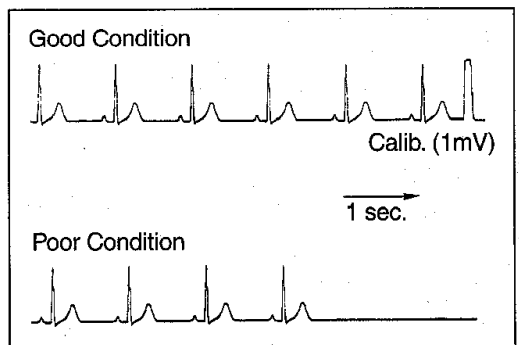

11. Results of digital ECG transmission in intelligent mode. transmitter and the output signal from the receiver was less than $5 \mathrm{sec}$, which is acceptable for the purpose of emergency care. However, if the communication link was cut for a relatively long period of time (more than several tens of seconds in our experiment), it took some time to resume data transmission. If the new communication link was good, it took 10-30 sec; but it took several tens of seconds or even several minutes if the new communication link was poor. This long delay was due to the time required to establish the communication protocol between the transmitting and receiving modems, which automatically select the parameters of data transmission according to the conditions of the communication link. This initial handshake takes a relatively long time (usually several tens of seconds).

Figure 12 shows a comparison of the two modes of data transmission. With digital ECG transmission, the correlation coefficient between the input and output waveforms is generally 0 or 1 . Thus, the number of received intact ECG wavelets was used as an indicator to represent the fidelity of the transmission. In the experiment, each ECG wavelet (from P-wave to $T$-wave) was sent every $1 \mathrm{sec}$ from an ECG simulator, and the received wavelets were counted every $10 \mathrm{sec}$. Therefore, fidelity of

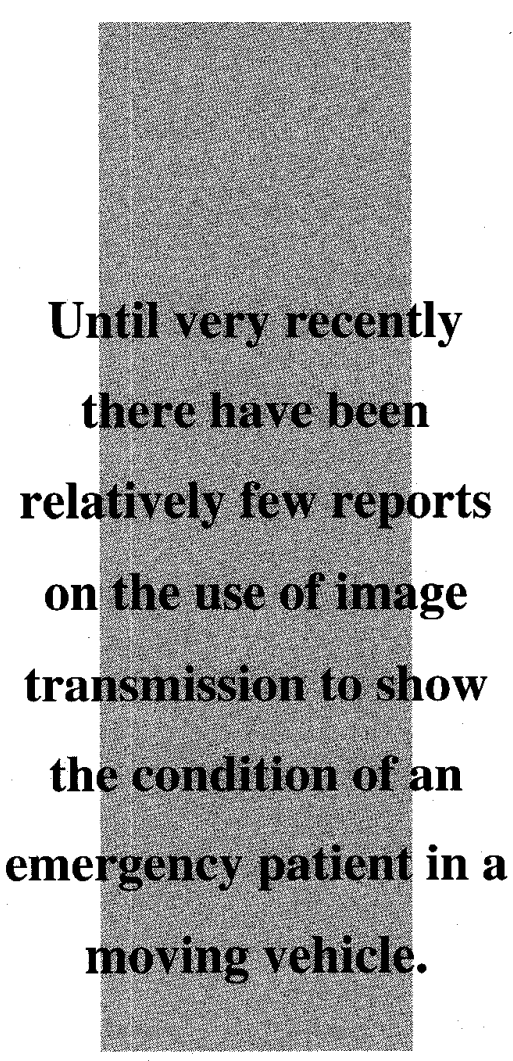

10 indicates perfect transmission. The results in Fig. 12 show a great improvement from the error-free mode to the intelligent mode. In the latter case, almost perfect

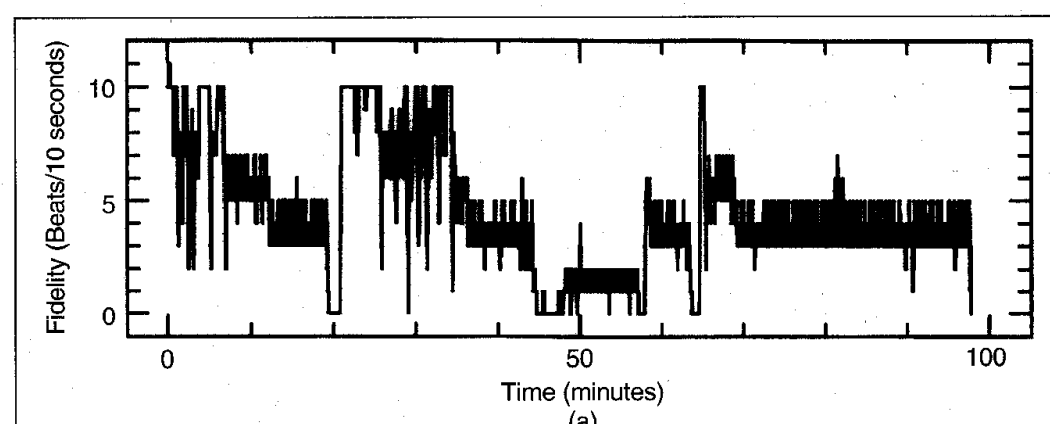

(a)

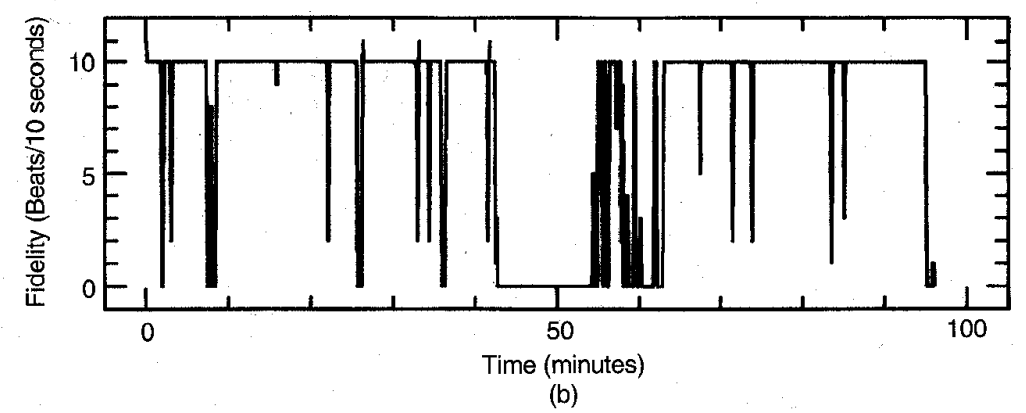

12. Temporal changes in the quality of received signals in digital ECG transmission: (a) in error-free mode, (b) in intelligent mode. 


\section{This application of}

mobile communication

can also bring about a

methodological change

in the concept of

conventional

telemedicine by

changing it from static

to dynamic. our city (Sapporo). The results of the experiments showed the characteristics of analog and digital ECG transmissions. In analog ECG transmission, the fidelity of data transmission varied with changes in the received radio wave. While in digital ECG transmission, the fidelity remained high until the strength of the radio wave dropped below a certain level. These characteristics can also be seen from a comparison of.Figs. 14(a) and (b).

Generally, if the correlation coefficient is more than 0.8 , we can identify the ECG waveform from background noises and recognize a change in heart rate by visual observation. Therefore, when the conditions of the communication link are not stable, such as in an emergency radio link, analog ECG transmission seems to be better. However, if the communication link is relatively stable, such as in a mobile telephone network, digital ECG transmission in the intelligent mode is more effective.

\section{Transmission of Color Images}

Although the need for ECG transmission from an ambulance has been widely recognized, until very recently there have been relatively few reports on the use of image transmission to show the condition of an emergency patient. The reason for this is the technical difficulties of practical image transmission in emergency cases. We have realized the significance of visual information for diagnosis by a medical doctor, and we have applied the image-transmission technique to mobile communication $[8,18-20]$.

Transmission experiments from a moving car were conducted using the system described above. The images transmitted were color still-pictures renewed every 0.5-1 min. First, an appropriate data-transmission rate was investigated. The rates examined were $2.4,4.8,7.2$, and $9.6 \mathrm{kbps}$. Using a higher rate $(7.2$ or 9.6 kbps), the image renewal time can be shortened (i.e., 20-30 sec) but the transmission becomes more susceptible to noise and vibration of the car. Based on the results of repeated transmission experiments, a transmission rate of $4.8 \mathrm{kbps}$ was selected as the most appropriate for our system. At this rate, data transmission remained reasonably stable, and images were renewed every $40 \mathrm{sec}$ on average. Figure 15 shows the color image transmitted from a moving car under stable condi-

transmission was attained if the conditions of the communication link were reasonable. However, if the conditions were relatively poor, the transmission was stopped and it took some time to resume.

Thus, it was shown that three-channel ECG transmission is feasible with a mobile telephone line in practical conditions. To test the usefulness of this technique, a three-channel ECG transmission was conducted from a moving ambulance to the emergency room of a hospital. This transmission was evaluated as "sufficiently useful" by a medical doctor specializing in emergency medicine.

\section{Comparison of ECG Transmissions}

To study the merits of analog and digital ECG transmissions, their characteristics were compared in transmission experiments from a moving car. Figure 13 shows the temporal variation of transmission fidelity with changes in the strength of the received radio wave. Fig. 14 shows the relationship between these two parameters. The routes taken by the car were almost the same in the analog and digital ECG transmission experiments, and the routes were confined to the central area of

\begin{tabular}{|l|l|l|l|}
\hline \multicolumn{5}{|c|}{ Table 3. Major Specifications of Mobile Stations } \\
\hline & Ship Station & Aircraft Station & Automobile Station \\
\hline Frequencies & $1.6 / 1.5 \mathrm{GHz}$ & $1.6 / 1.5 \mathrm{GHz}$ & $1.6 / 1.5 \mathrm{GHz}$ \\
\hline Antenna & Short back-fire & Phased array & TBD \\
\hline $\mathrm{G} / \mathrm{T}$ & $-10 \mathrm{~dB} / \mathrm{K}$ & $-12 \mathrm{~dB} / \mathrm{K}$ & $-15 \sim-18 \mathrm{~dB} / \mathrm{K}$ \\
\hline Max. TX. Power & 70 watts & 100 watts & $10 \sim 20$ watts \\
\hline Max. EIRP & $32 \mathrm{dBW}$ & $29 \mathrm{dBW}$ & $20 \mathrm{dBW}$ \\
\hline MODEMs & $\begin{array}{l}\text { MSK }(16 \mathrm{k} / 24 \mathrm{k}) \\
\text { NBFM, PBSK }(4.8 \mathrm{k}) \\
\text { BPSK }(160 \mathrm{k})\end{array}$ & $\begin{array}{l}\text { MSK }(24 \mathrm{k}), \mathrm{NBFM}(4.8 \mathrm{k}) \\
\text { BPS, }\end{array}$ & $\begin{array}{l}\text { ACSB } \\
(\text { low-speed digital) }\end{array}$ \\
\hline CODECs & CADM, ADM, MPC & CADM, ADM & TBD \\
\hline
\end{tabular}

Table 4. Major Specifications of On-Board Transponders and Antennas

\begin{tabular}{|l|l|l|}
\hline & L-Band & C-Band \\
\hline Antenna & Offset parabola & Horn \\
\hline $\mathrm{G} / \mathrm{T}$ & $-3 \mathrm{~dB} / \mathrm{K}$ & $-8 \mathrm{~dB} / \mathrm{K}$ \\
\hline Max. TX. Power & 25 watts & 7 watts \\
\hline Max. EIRP & $35.5 \mathrm{dBW}$ & $24.5 \mathrm{dBW}$ \\
\hline Bandwidth & $3 \mathrm{MHz}$ (except L/L link) \\
\hline Weight & $65 \mathrm{~kg}$ (including antennas) \\
\hline Power Consumption & 300 watts \\
\hline
\end{tabular}


tions; i.e., traveling at a speed of about 50 $\mathrm{km} / \mathrm{h}$ on an asphalt-surface road. As can be seen in the figure, the transmitted image was of sufficient quality for understanding the patient's condition.

For comparison with extreme conditions, a color image was transmitted from the car traveling on a gravel road in a mountainous area. The vibrations of the car were so strong that the experimenters could not remain standing. The image was disturbed to some extent by noise, which appeared as white horizontal lines and as a change in the colors in a horizontal band. However, despite the noise, the quality of the images was still sufficient for understanding the patient's condition.

To examine the practical usefulness of this technique, transmission experiments were conducted under various conditions that were likely to be encountered in emergency cases. In the city area, the ECG and images were transmitted from various points; e.g., from a street with many tall buildings, from a narrow road between two tall buildings, under the overhead lines of streetcars, under overhead train lines, and from an underground parking lot. In the suburbs, signals were transmitted from residential areas, from bighways, and from hilly areas. No prob-

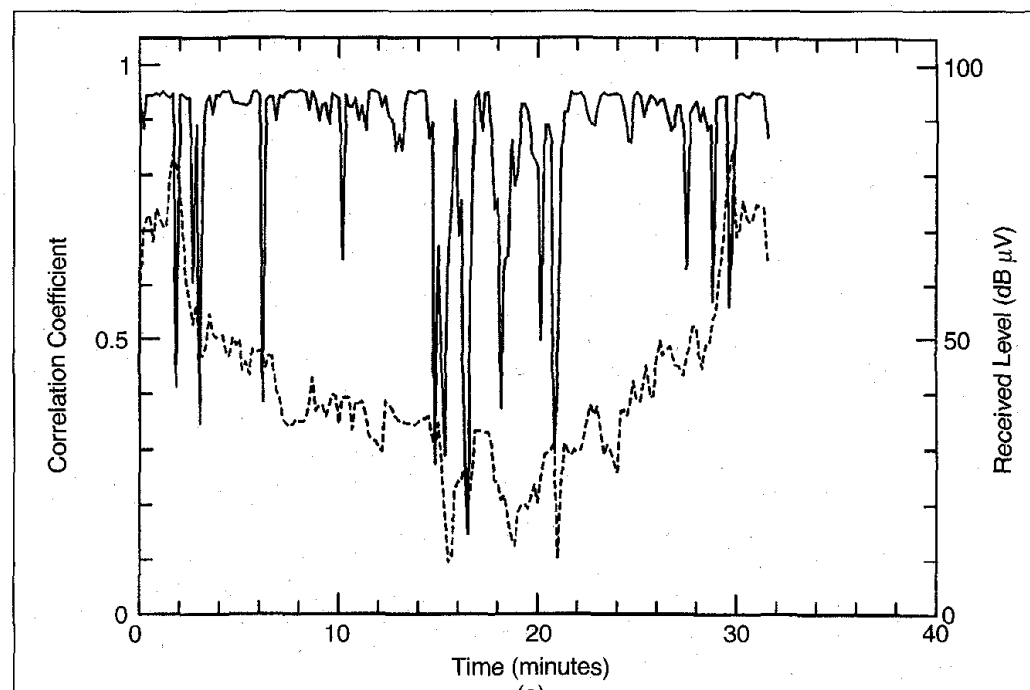

(a)

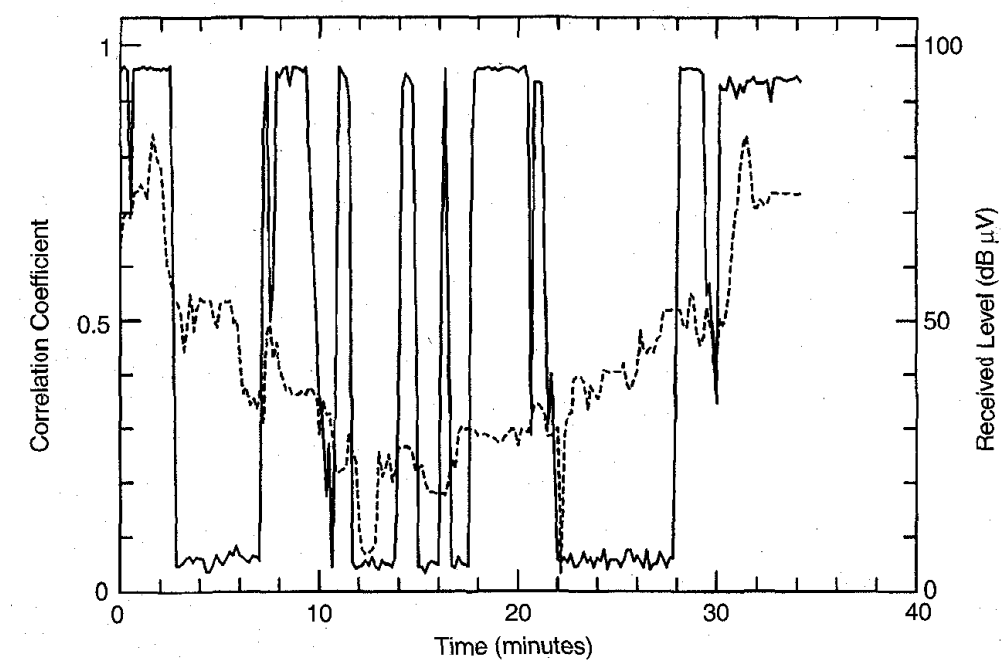

(b)

13. Comparison of analog and digital ECG transmissions using temporal changes of correlation coefficient (solid line) and received level of radio wave (dashed line): (a) analog ECG transmission, (b) digital ECG transmission. lems for practical use were found in most of these locations. The only problems found were some long lapses in the communication link when the ECG and images were transmitted from underground parking lots or from behind a high mountain. The experiments were repeated using a mobile telephone line and an emergency radio link. With the emergency radio link, the communication link was relatively unstable and noise was greater than that in the mobile telephone line. However, except for this difference, the results obtained using these two communication links were almost the same.

To evaluate the practicality of this system, an experiment was conducted on simultaneous transmission of ECGs and color images from a moving ambulance to the emergency room of a hospital. A medical doctor specializing in emergency medicine evaluated the quality of the received signal, and he found it satisfactory for emergency practices.

\section{Telemedicine via Sutellite Communication Satellite Link}

The satellite link was developed with the cooperation of the Communications Research Laboratory of the Ministry of Posts and Telecommunications, the Electronic Navigation Research Institute of the Ministry of Transport, and the National Space Development Agency of Japan. Mobile stations have been developed for installation in ships, aircraft, and automobiles. The ship station is small enough to be installed in a vessel of about 30 tons. The weight of above-deck equipment with a $40 \mathrm{~cm}$ short backfire antenna is about $45 \mathrm{~kg}$. Countermeasures were taken against the fading effect caused by sea surface reflection. The major specifications are shown in Table 3.

The aircraft station was designed to be installed on B-747 cargo planes. The antenna system was designed to be mounted inside the roof. The frequency shift due to the Doppler effect was compensated for. The major specifications are shown in Table 3 .

The ETS-V satellite is a three-axis stabilized geostationary satellite. On-board equipment for communication experiments consists of L-band and C-band antennas and transponders. The major specifications are shown in Table 4 . The fixed station, which has RF equipment and antennas for C-band and L-band links, was set up in a laboratory on the 


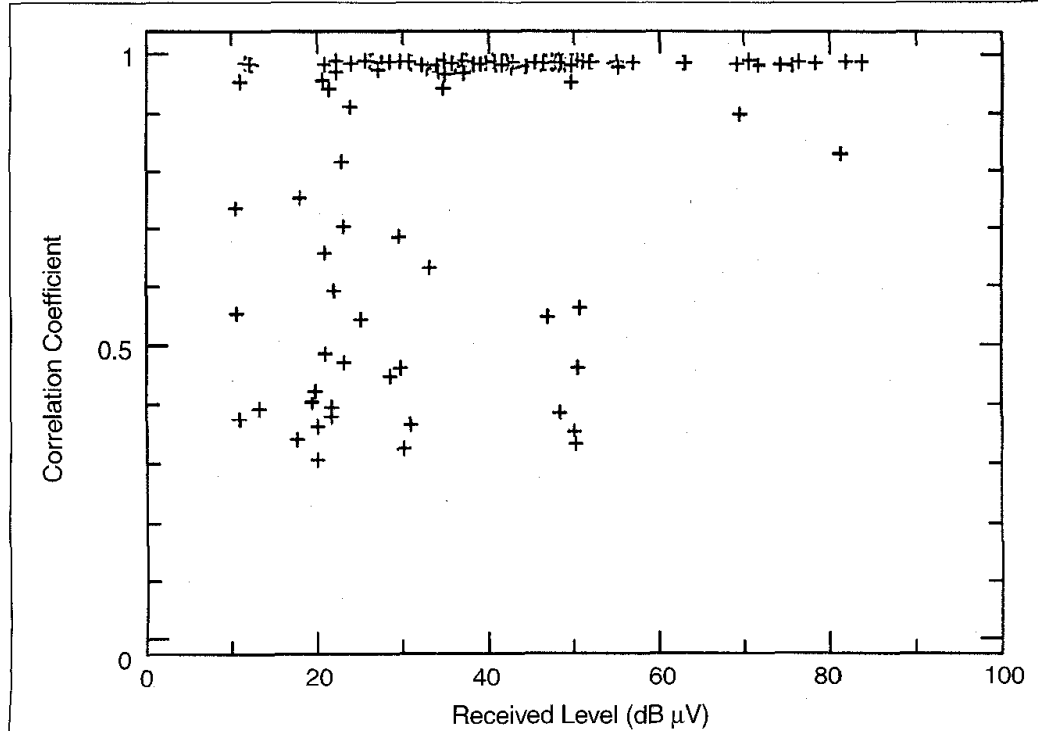

(a)

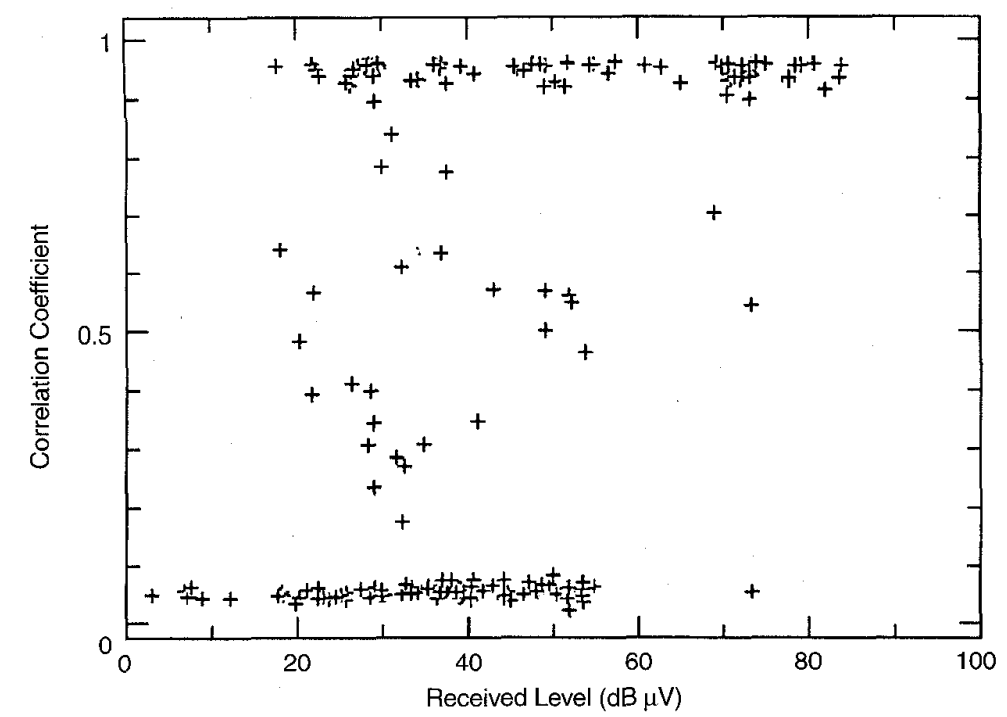

(b)

14. Comparison of analog and digital ECG transmissions in the relationship between correlation coefficient and received level of radio wave: (a) analog ECG transmission, (b) digital ECG transmission.

ground. The major specifications of the fixed station are shown in Table 5.

\section{Medical Data-Transmission System}

Figure 16 shows the outline of the medical data-transmission system. The data flow from the mobile station to the fixed station is as follows. In the vehicle shown in Fig. 16(a), the audio, ECG (three channels), and blood pressure signals are acquired at specified intervals from the patient and are encoded in a personal computer. The image signals are encoded by the image-compression system, and the puter. The image signals and other signals are multiplexed in time and fed to the modulator of the satellite link. The multiplexed data is sent to the satellite in a serial mode and transferred to the fixed

In the ground station, shown in Fig.16(b), the signals are obtained from the demodulator of the satellite link and are demultiplexed in a personal computer. The image signals are sent to the image coded signals are sent to a personal comground station. decompression system, and the picture is decoded and displayed. Other signals are directly decoded in the personal computer to produce the outputs. If an error in an ECG signal is detected in the decoding process, an ARQ signal is generated. The error correction of blood pressure signals is executed in the personal computer.

The data flow from the fixed station to the mobile station is as follows. In the fixed station (Fig.16(b)), the voice of the doctor is encoded in a personal computer. The encoded signals are multiplexed with the ARQ signals from the ECG error detector and then sent to the modulator of the satellite link. The signals are sent to the satellite in a serial mode and transferred to the mobile station.

In the mobile station (Fig.16(a)), the signals from the demodulator are separated and decoded. The audio signals are directly decoded to an audio output. ECG ARQ signals are sent to the encoder in the personal computer. The stored corresponding data are then transferred to the transmitter buffer. Automatic retransmission is achieved by transmitting these data again to the fixed station through the satellite link.

\section{Transmission from a Navigating Ship}

After the basic performance of the proposed system was verified by experiments in a fixed station, a transmission experiment from a navigating ship was conducted. The mobile station was installed in the training ship (1400 tons) of our university. The fixed station was set up in the ground station of the Communications Research Laboratory.

In the experiment, a variable noise was added immediately before the demodulator of the satellite link to change the state of the communication link. The color image, audio signal, ECG, and blood pressure were transmitted separately, and the quality of the data after decoding was examined. The lower limit of the carrier-to-noise ratio $\left(\mathrm{C} / \mathrm{N}_{0}\right)$ that would guarantee satisfactory quality of transmission for telemedicine was investigated for each type of information. Figure 17 shows some examples of the received images. When $\mathrm{C} / \mathrm{N}_{0}$ was $55.1 \mathrm{dBHz}$ (Fig. 17 (b)), the shape of the object was clear and color reproduction was good in more than half of the picture area. As $C / \mathrm{N}_{0}$ decreased further, errors in the image increased rapidly. Thus, the threshold value for image quality was determined to be $55.1 \mathrm{dBHz}$. 
Table 6 summarizes the results of the experiments. The reliability of blood pressure transmission was improved by applying the error-correction technique, and satisfactory transmission was achieved up to $49.9 \mathrm{dBHz}$. This agrees with the results of the theoretical analysis (Fig. 2).

The transmission experiments were conducted from different points over a period of two weeks while the ship navigated along the coast of Japan. During this period, the conditions of the satellite link were generally favorable. However, communication was occasionally interrupted when the ship was oriented in a certain direction. The cause of this was found to be that the main mast of the ship blocked the main lobe of the antenna, which was installed close to the base of the mast. This problem can be solved by setting the antenna at an appropriate position on the ship. Except for this problem, we could not find any significant difficulty in providing telemedicine on a navigating ship. A detailed description of the characteristics of the satellite link with the ship station can be found elsewhere [21, 22].

\section{Transmission from an Aircraft in Flight}

A transmission experiment was also conducted from an aircraft in flight. The mobile station was installed in a jet cargo plane (Boeing 747), and the fixed station was set up in a ground station. The flight route was along the great circle from Narita, Japan (New Tokyo International Airport) to Anchorage, Alaska, USA. The transmission experiment was conducted in the same manner as that for the navigating ship. Figure 18 shows examples of transmitted images. Again, when the $\mathrm{C} / \mathrm{N}_{0}$ of the satellite link was more than 55 $\mathrm{dBHz}$, satisfactory quality was obtained in the separate transmission of image, sound, ECG, and blood pressure.

A simultaneous transmission of image, sound, ECG, and blood pressure was attempted. With $\mathrm{C} / \mathrm{N}_{0}$ of more than 55 $\mathrm{dBHz}$, good multiplexing and demultiplexing without any crosstalk were confirmed. In this experiment, change in the $\mathrm{C} / \mathrm{N}_{0}$ of the satellite link was less than several $\mathrm{dBHz}$ during an approximately 20-hour round-trip flight from Narita, Japan, to Anchorage, USA. The communication link was not blocked as it was in the case of the ship, and the feasibility of telemedicine in a flying aircraft was verified. Detailed descriptions of

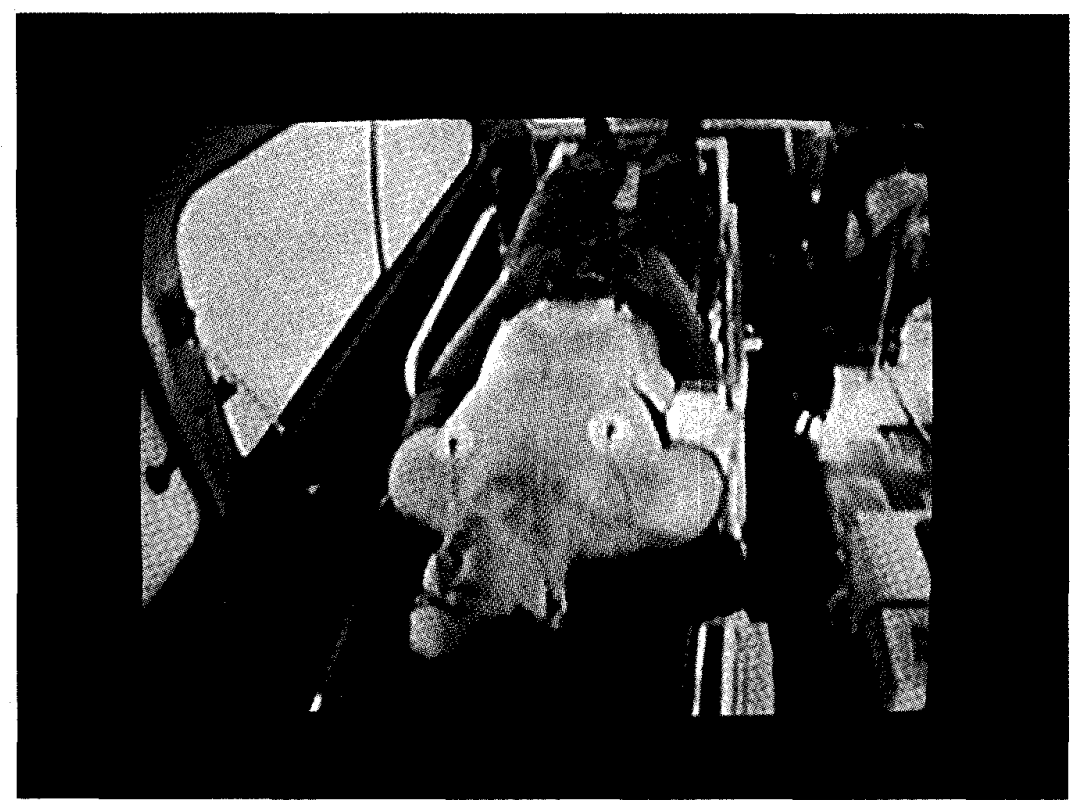

15. An example of a color image transmitted from a moving car.

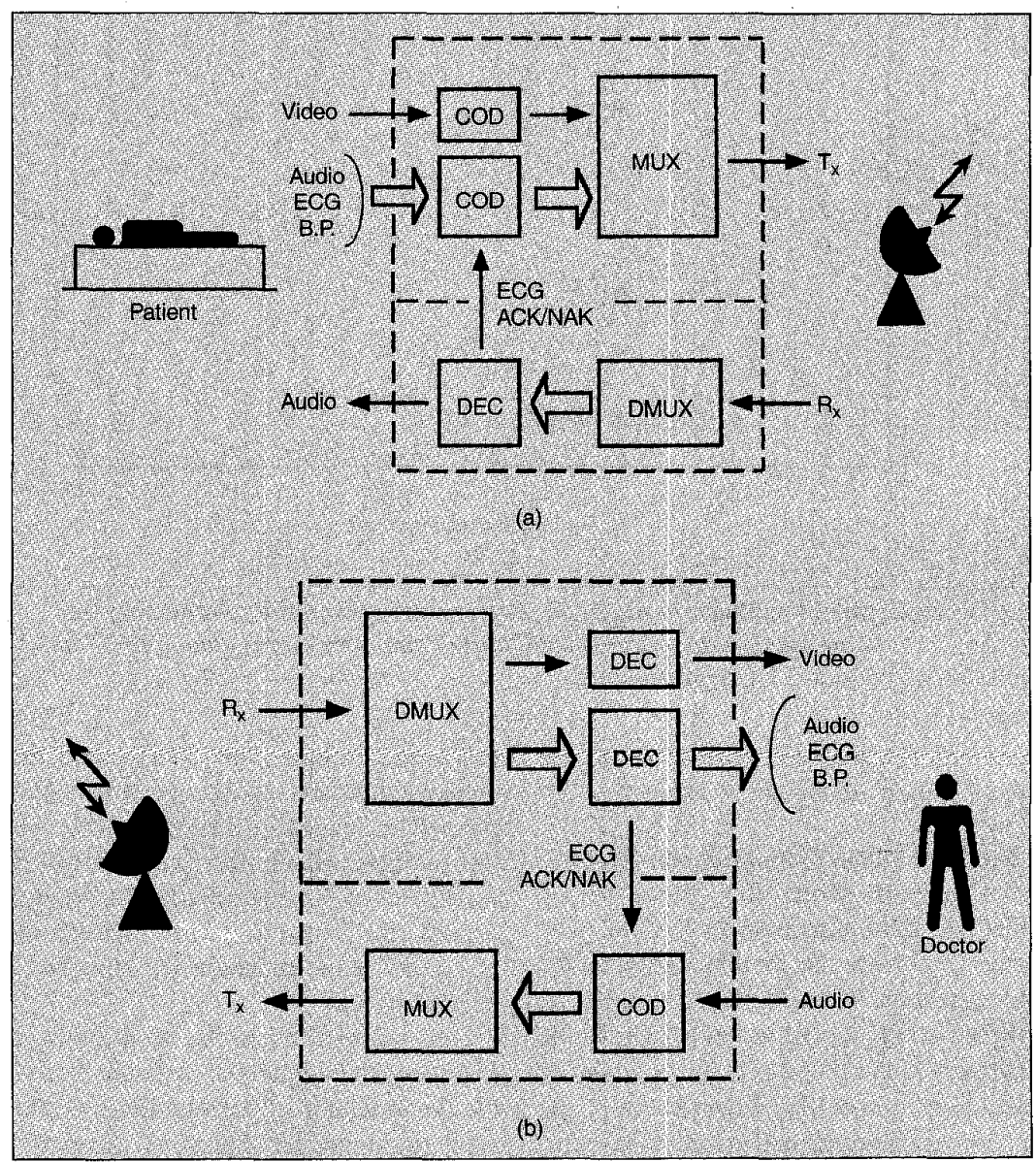

16. Outline of data flow in the developed system: (a) Mobile station, (b) fixed station.

IEEE ENGINEERING IN MEDICINE AND BIOLOGY 


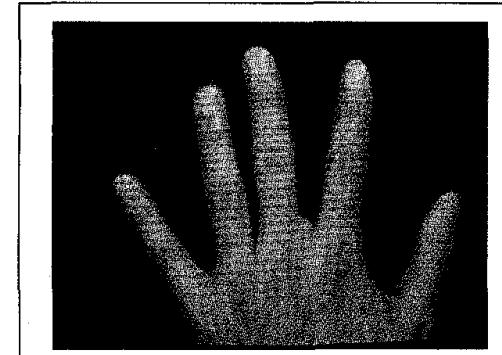

(a)

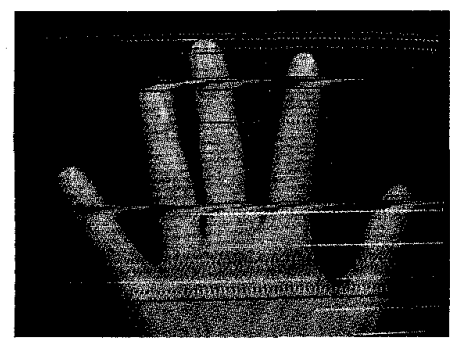

(b)

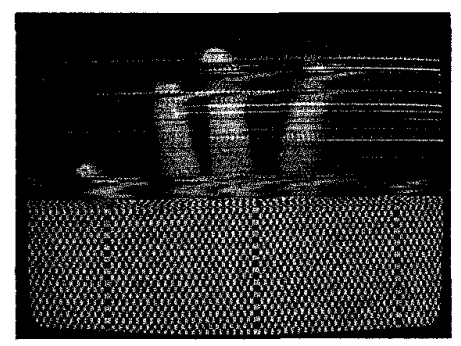

(c)

17. Color images transmitted from a navigating ship to a fixed station: (a) $\mathrm{C} / \mathrm{N}_{\mathbf{0}}=\mathbf{6 5 . 5} \mathrm{dBHz}$, (b) $\mathrm{C} / \mathrm{N}_{0}=55.1 \mathrm{dBHz}$, (c) $\mathrm{C} / \mathrm{N}_{0}=53.1 \mathrm{dBHz}$.

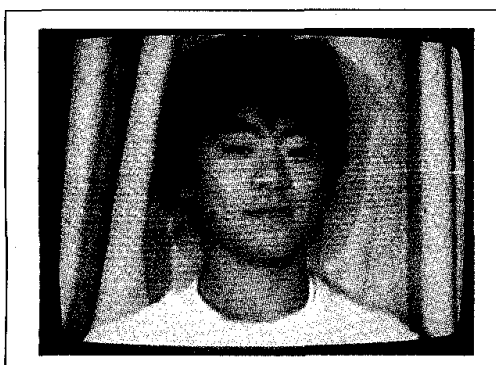

(a)

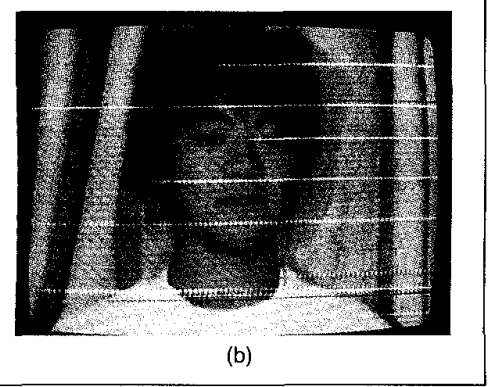

18. Color images transmitted from a flying aircraft to a fixed station: (a) $\mathrm{C} / \mathrm{N}_{0}=58.9 \mathrm{dBHz}$, (b) $\mathrm{C} / \mathrm{N}_{0}=54.0 \mathrm{dBHz}$.

\begin{tabular}{|l|l|l|l|}
\hline \multicolumn{3}{|l}{ Table 5. Major Specifications of Fixed Station (Kashima, Japan) } \\
\hline \multirow{3}{*}{ Frequencies } & C-Band & L-Band \\
\hline & Transmitting & $5960+11.25 \mathrm{MHz}$ & $1646.25+3.75 \mathrm{MHz}$ \\
\cline { 2 - 4 } & Receiving & $5230+11.25 \mathrm{MHz}$ & $1544.25+3.75 \mathrm{MHz}$ \\
\cline { 2 - 4 } & TT\&C & $\begin{array}{l}5105 \mathrm{MHz} / \\
6396 \mathrm{MHz}\end{array}$ & \\
\hline Antenna & & Cassegrain $10 \mathrm{~m}$ & Parabola $3 \mathrm{~m}$ \\
\hline G/T & & $31 \mathrm{~dB} / \mathrm{K}$ & $6 \mathrm{~dB} / \mathrm{K}$ \\
\hline Max. Tx. Power & & $300 \mathrm{watts}$ & 30 watts \\
\hline Max. EIRP & & $78 \mathrm{dBW}$ & $44 \mathrm{dBW}$ \\
\hline
\end{tabular}

\begin{tabular}{|l|c|c|}
\hline \multicolumn{2}{|c|}{ Table 6. CNo Thresholds in Data Transmission (dBHz) } \\
\hline Data & Nonerror Transmission & Permissible Transmission \\
\hline Video & 57.1 & 55.1 \\
\hline Audio & 56.9 & 55.0 \\
\hline ECG & 55.0 & \\
\hline Blood Pressure & 49.9 & \\
\hline
\end{tabular}

these experiments can be found elsewhere $[8,23,24]$.

\section{Summary and Conclusions}

A concept of mobile telemedicine has been proposed to provide emergency care in a moving vehicle. The practicality of this technique was investigated through technical considerations required to realize mobile telemedicine. Some problems with this technique were identified, and measures to resolve the problems were devised. Then, theoretical analysis verified the feasibility of the proposed technique. Different multiplexing techniques for the multiple medical data transmission by mobile communication were investigated. An experimental system that can simultaneously transmit color images, an audio signal, three-channel ECGs, and blood pressure from a moving vehicle to a fixed station was developed. Experiments on the transmission of multichannel medical data from a moving ambulance, a navigating ship, and a flying aircraft were conducted. The results of these experiments verified the practical feasibility of the proposed technique.

In the practical application of this technique, there may be some legal problems: for example, whether medical treatment through a communication medium would be legally acceptable or not, and whether the transmission of medical data violates the protection of personal privacy. However, considering the emergency nature of this technique and the significance of the results, both problems seem to be either legally or technically solvable.

This application of mobile communication to telemedicine is not confined to merely a proposal to use new techniques. It can also bring about a methodological change in the concept of conventional telemedicine by changing it from static to dynamic, and by enlarging its scope from a local area to a global or cosmic area. It may also have an impact on conventional emergency medicine in that it will open up a new field of application that applies to moving vehicles.

\section{Acknowledgments}

The author wishes to thank the staff of the Communications Research Laboratory, Ministry of Posts and Telecommunications, and the staff of the Bureau of Telecommunication for their cooperation in the experiments. The author is also grateful to Professor Goro Matsumoto, Hokkaido University; Professor Tomohisa Mikami, Hokkaido University; Professor Nozomu Hoshimiya, Tohoku University; Professor Takeshi Hatsuda, Hokkaido Institute of Technology; and Professor Katsuyuki Yamamoto, Hokkaido University for their valuable advice. 
A part of this research was supported by a grant from the Ministry of Education, Science, Sports, and Culture, Japan.

Koichi Shimizu was born in Otaru, Japan, in 1950. He received the B.S. from Hokkaido University, Sapporo, Japan, in 1973. In 1976 and 1979 he received the M.S. and the Ph.D., respectively, from the University of Washington, Seattle. From 1974 to 1979 he was a research associate in the Center for Bioengineering and the Department of Electrical Engineering, University of Washington, Seattle. From 1979 to 1987 , he was an instructor at the Research Institute of Applied Electricity, Hokkaido University, Sapporo, Japan. From 1987 to 1995 , he was an instructor and an associate professor at the Faculty of Engineering, Hokkaido University, Sapporo, Japan. He is currently a professor at the Graduate School of Engineering, Hokkaido University, Sapporo, Japan. He also serves as a professor at the Graduate School of Engineering, Tohoku University, Sendai, Japan. He has been engaged in the studies of biomedical engineering including those of wave propagation in biological media, biotelemetry, and biological effects of electromagnetic field. He is a member of IEEE; the International Society on Biotelemetry; the Institute of Electronics, Information and Communication Engineers of Japan; the Institute of Electrical Engineers of Japan; and the Japan Society of Medical Electronics and Biological Engineering.

Address for Corrrespondence: Koichi Shimizu, Department of Biological Systems Engineering, Graduate School of En- gineering, Hokkaido University, Sapporo, 060 Japan. Tel: +81-11-706-6857. Fax: +81-11-706-7844. E-mail: shimizu@ bme.eng.hokudai.ac.jp.

\section{References}

1. Mackay RS: Bio-Medical Telemetry. IEEE Press, Piscataway NJ, 1993.

2. Allan R: Coming: The era of telemedicine. IEEE Spectrum 13(12): 30-35, 1976

3. Elford DR: Telemedicine in northern Norway. J Telemedicine and Telecare 3(1):1-22, 1997.

4. Berteand M, Guardo R, Roberge FA, Blondeau P: Microprocessor application for numerical ECG encoding and transmission. Proc IEEE 65:714-722, 1977.

5. Uhley HN: Electrocardiographic telemetry from ambulances - A practical approach to mobile coronary care units. Am Heart $J$ 80:838-842, 1970.

6. Grim P, Feldman T, Martin M, Donovan R, Nevins V, et al.: Cellular telephone transmission of 12-lead electrocardiograms from ambulance to hospital. Am J Cardiol 60:715-720, 1987.

7. Moller J: Health care via satellite. Computer Graphics World 69-70, June 1988.

8. Murakami H, Shimizu K, Yamamoto K, Mikami T, Hoshimiya N, Kondo K: Telemedicine using mobile satellite communication. IEEE Trans Biomed Eng 41:488-497, 1994.

9. Kohli J: Medical imaging applications of emerging broadband networks. IEEE Commun Mag (27):8-16, Dec. 1989.

10. Yamaguchi H: Vector quantization of differential luminance and chrominance signals. IEEE Trans Commun COM-33:457-464, 1985.

11. Jayant NS: Adaptive delta modulation with a one-bit memory. Bell Syst Tech J 49:321-342, 1970

12. Jayant NS: Digital coding of speech waveforms. Proc IEEE 62:611-632, 1974

13. Jalaleddine SMS, Hutchens CG, Strattan RD Coberly WA: ECG data compression tech- niques - a unified approach. IEEE Trans Biomed Eng BME-37:329-343, 1990

14. Kudo N, Shimizu K, Matsumoto G: Optical biotelemetry using indirect light transmission. Biotelemetry 9:91-98, 1987.

15. Shimizu K: Optical biotelemetry. Jap J Physical Fitness and Sports Medicine 44:317-324, 1995.

16. Matsuda S, Shimizu K, Yamamoto K: Multiplexing technique of audio and medical signals for emergency radio. Jap J Med Elec Bio Eng 31:74-78, 1993 .

17. Murakami $\mathbf{H}$, Shimizu $\mathbf{K}$, Yamamoto $\mathbf{K}$, Mikami T, Hoshimiya N, Kondo K: Medical data transmission using mobile satellite communication. Trans IEICE J72-B-II: 262-268, 1989.

18. Shimizu K, Matsuda S, Saito I, Yamamoto K, Hatsuda T: Application of biotelemetry technique for advanced emergency radio system. IEICE Trans Commun E78-B:818-825, 1995.

19. Shimizu K, Murakami H, Yamamoto $K$, Mikami T, Hoshimiya N, et al: Multiple medical data transmission from mobile station using ETS-V. Rev Comm Res Lab 36(10):105-111, 1990.

20. Shimizu K, Murakami H, Yamamoto $K$, Mikami T, Hoshimiya N, et al.: Medical telemetry from moving vehicles using a communication satellite. Biotelemetry 11:242-245, 1991.

21. Ikegami T, Wakana H, Kawamata F, Ide T, Matsumoto Y: Experiments on maritime satellite communications using ETS-V. Rev Commun Res Lab 36(10):47-56, 1990.

22. Sakasai M, Arakaki Y, Morikawa $E$, Matsumoto Y: Experimental results of TDM/TDMA system via ETS-V. Rev Commun Res Lab 36(10):57-62, 1990.

23. Taira S, Ohmori S, Tanaka M: High gain airborne antenna for satellite communications. Rev Commun Res Lab 36(10):39-46, 1990.

24. Ohmori S, Hase Y, Wakana H, Taira S, Ide T: Experiments on aeronautical satellite communications using ETS-V. Rev Commun Res Lab 36(10):27-37, 1990 